\title{
Enhancement of Data Aggregation Grid based Coverage Ratio using Overlap Sensing Ratio with Awgn Channel in Heterogeneous WSNs
}

\author{
Akansha Elisha George \\ M. Tech Scholar \\ ECE Department \\ SHIATS-DU \\ Allahabad, U.P., India
}

\author{
Rajeev Paulus \\ Assistant Professor \\ ECE Department \\ SHIATS-DU \\ Allahabad, U.P., India
}

\author{
A.K. Jaiswal \\ Professor (H.O.D.) \\ ECE Department \\ SHIATS-DU \\ Allahabad, U.P., India
}

\begin{abstract}
Heterogeneous wireless sensor network (H-WSN) consists of sensor nodes with different ability, such as different computing power and sensing range. In this Research paper, the improved - enhance coverage overlapping sensing ratio (IECOSR) scheme for data aggregation in grid based WSNs is proposed. Here, the focus was on two main problems i.e. in the area coverage problem, the coverage performance of the covered region was enhanced while in the dead node coverage problem; the coverage performance on the number of covered area by maximum efficient energy node for heterogeneous network with different communication and sensing range. It is based on the random wave point sensor model used to approximate the behavior of sensor nodes. According to experimental results, the proposed method can achieve higher coverage and sensing range for the position of dead node by shifting a higher energy node in one grid in order to overlap the lower energy node in other grid which comes under the sensing range of the advanced node which can be improving network lifetime \& recover data loss crises for data aggregation technique by using I-ECOSR with AWGN channel, which is simulated in MATLAB 2012a.
\end{abstract}

\section{Keywords}

H-WSN, sensing ratio, directional sensor nodes, AWGN, Node Energy.

\section{INTRODUCTION}

In the area of wireless sensor networks (WSN), to successfully accomplish the allocated sensing tasks, the positioned must have to cover sensors area to confident specific particular point-locations or it can be said as complete mark sensing zone, \& method a connected network through multi-hop wireless communications. Hence, positioning WSN to run associated coverage is a main research concern. In previous researches, the present solutions to the connected coverage issue in WSN accept Omni-directional sensors with dish like sensing range [1], [2], [3]. Though due to equipment limitations or environmental impairments, positive sensors are directive and only facilitate a sector-like sensing range, which are called directional sensors.

\subsection{The Sensing coverage}

The Sensing coverage is an important issue in WSNs. It shows how to properly maintain an environment \& monitor, \& works on the base of real applications such as target detection, physical phenomenon and follow the target location. Because of the heterogeneity of the sensor networks, the concept of sensing coverage results to an extensive range of apprehension. However, only the individual isotropic sensors have been studied in the previous works. For example, most of the previous study shows that in point coverage problems (e.g. [1]-[5]), the sensing capacity of sensors is introduced as a circular region (or disk) \& an occurrence or goal sensors is sensed in a second sense that depends on whether it is confidential which is sensing disk or not. On the basis of comparison to isotropic sensors, the coverage area of a directional sensor is resoluted by both its position \& direction. It can be superlative demonstrated by the example presented in Figure 1.

In this paper, goal was to identify \& resolve the issues of coverage ratio by random directional sensors with tunable (able to tuned or modified) orientations under the random deployment approach. It can improve a solution that maximizes the no. of aims to be covered while reducing the no. of sensors to be stimulated at any instance. Also the current solutions contain sleep development for the nodes.
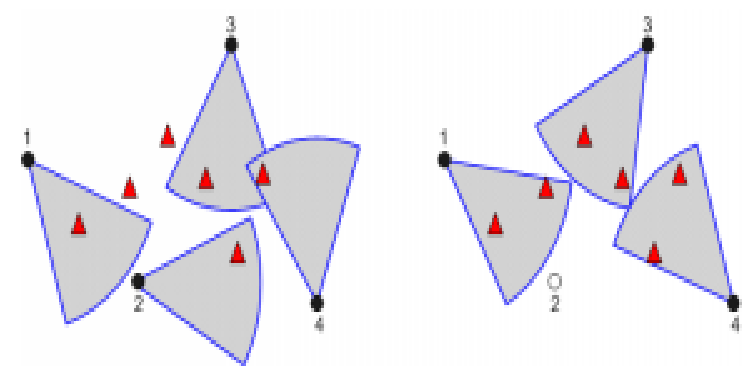

Fig 1: in above figure 2 norms are possible of 4 directional sensor nodes (black circles) positioned to cover 6 targets (red triangles) in a sensor field. [Left] Case I: all 4 nodes are dynamic active whereas 2 targets are not covered

[right] Case II: 3 nodes are active with no target.

As compared deterministic positioning, the random deployment, is easy and less expensive for large wireless sensor networks, and may be the only possible option in remote or in hospitable environments. Furthermore, to compensate for the absence of exact standing and progress the fault tolerance, nodes are classically positioned in excess, \& thus terminated sensors frequently arise. Moreover, sensors are commonly power-driven by battery and it may not be possible to recharge or switch the batteries once positioning. In calculation, target locations may change even after first deployment, thus changing the optimal solution to the coverage problem.

\subsection{Network lifetime}

In wireless network, mostly the sensor nodes suffer due to its partial battery power volume. Because of the meager size as of present batteries, those sensor nodes do not last as extensive as preferred. Then directional sensor nodes have several working directions, rotatable mechanical design is 
required to utilize all working directions. Physical movement consumes definitely much more power than other activities [3]. Therefore, physical activities like rotating a sensor node around its axes or moving it to another position should be well planned to minimize the energy consumption.

\subsection{AWGN channel}

Additive white Gaussian noise (AWGN) channel is a noise model that deals with the imperfection caused by noise in the communication channel. The disturbance caused by the thermal noise due to the electron movement is generally modeled as AWGN.This noise channel is classically decent for cable and profound space communication but not in experienced communication because of multipath fading, land obstructive and interfering.

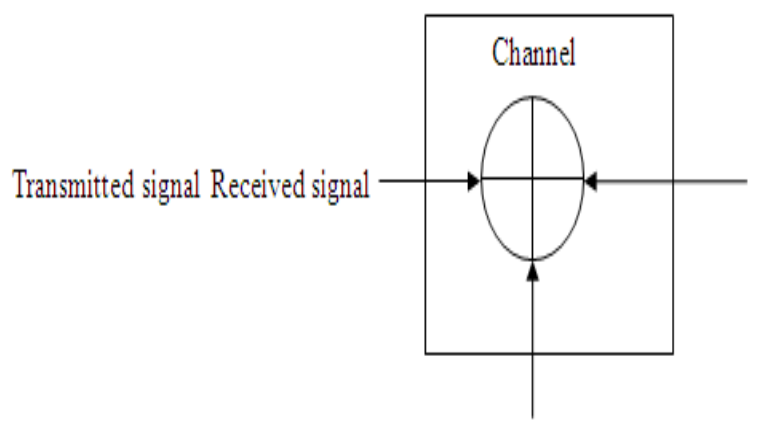

AWGNnoise (sound)

Fig:2

AWGN is used to represent contextual noise in channel. The term additive refers to the addition of noise in the transmitted signal in time domain. So, the received signal can be given as $\mathrm{R}(\mathrm{t})=\mathrm{S}(\mathrm{t})+\mathrm{N}(\mathrm{t}) \quad$ where, $\mathrm{S}(\mathrm{t})$ is a transmitted signal; while $\mathrm{N}(\mathrm{t})$ is contextual noise.

\section{PROBLEM STATEMENT}

Nowadays, in the field of directional sensor networks, nodes coverage is a big issue for the many researchers, in fact the problem arises not only in area of coverage but also the dead node occurrence in the network as well. The Area coverage problem is an important issue in directional sensor networks. This issue is to maximize the area coverage of a randomly positioned directional sensor network. Every directional sensor can rotate through orientation to grow improved coverage in a concerned area. Hence, the I-ECOSR algorithm is proposed to enhance the area coverage. This paper intent to maximize the area coverage of a randomly positioned directional sensor network. The problem of working direction forecast to cover best areas, called Maximum Directional Area Coverage (MDAC) problem. The directional sensing model is designed and systems for the Maximum Directional Area Coverage (MDAC) problem. In a directional sensor network, each directional sensor cannot sense the entire circular area.

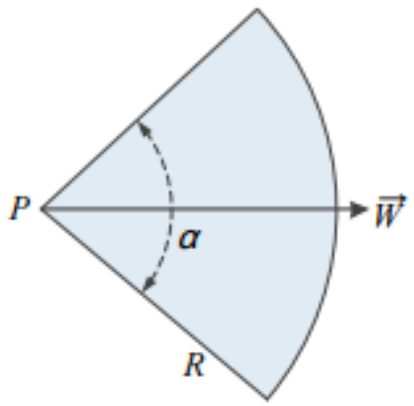

Fig 3: The directional sensing model of maximum directional area coverage

Demonstration of the directional sensors \& their characteristics:

1. In wireless sensor network each directional sensor is homogeneous, such as: sensing angle, sensing radius, $\&$ communication radius.

2. Every directional sensor can sense only one direction with restricted measurement.

3. All directional sensors are fixed and can rotate random angle in sensing region.

4. The communication radius is twice than the sensing radius such that sensing neighbors can dependably and efficiently communicate.

\section{SYSTEM MODEL}

\subsection{Sense the Random Direction of Region :}

- In this system model, the sensing field with nodes presence positioned randomly square area is used to define the square region Fig.4 (a).

- The bi-dimensional area is developed to model the sensing region of directional sensor, which is shown in Fig.4 (b).

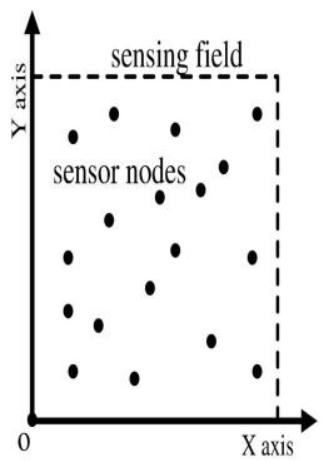

(a)

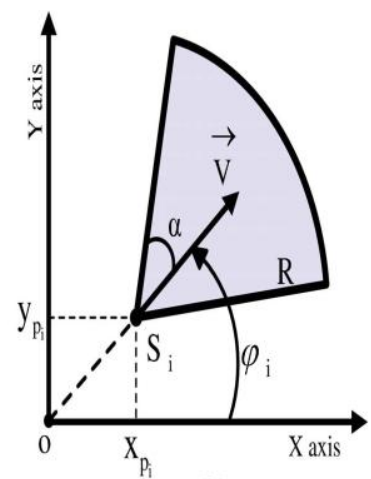

(b)
Fig 4: Sensing field and directional sensing field

The directional sensing model can be signified by $\left(\mathrm{P}_{\mathrm{i}}, \mathrm{R}, \mathrm{v}, \alpha\right)$ where $P_{i}(x, y)$ signifies the position of nodes $S_{i}, R$ is the radius of a sensing region that directs the maximum sensing range of nodes. $\rightarrow$ Is a unit vector called sensing direction that splits a sensing area into two parts. The Sensing position is represented by $\alpha$, where $2 \alpha$ defines the sensor's field of view (FOV). Intersection angle among the sensing direction of nodes $S_{\mathrm{i}}$ and $\mathrm{X}$ axis is called the direction angle indicated by $\varphi \mathrm{i}$ with the range of $[0,2 \Pi)$. In the sensing field, nearby sensors nodes whose Euclidean distance to the present node is less than $2 \mathrm{R}$ ? It is sufficient to deliberate the consequence of 
neighboring sensors and adjust node's sensing direction. It can be seen that $\mathrm{S} 1$ and $\mathrm{S} 2$ are the neighboring sensors of $\mathrm{S}_{\mathrm{i}}$, $\mathrm{S} 3$ is not the neighboring sensor because the distance between $\mathrm{S}_{\mathrm{i}}$ and $\mathrm{S} 3$ is larger than $2 \mathrm{R}$. The grid part signifies the overlapping section of $\mathrm{Si}$. The centroid of the overlapping region is represented by Cen. The centroid angle is the angle between $\mathrm{X}$ axis \& the line which pass finished Cen and the node, represented by $\beta_{i}$ with the range of $[0,2 \Pi)$ shown in Fig.3.Based on that diagram find the direction angle for rotating sensors to cover the maximum area.

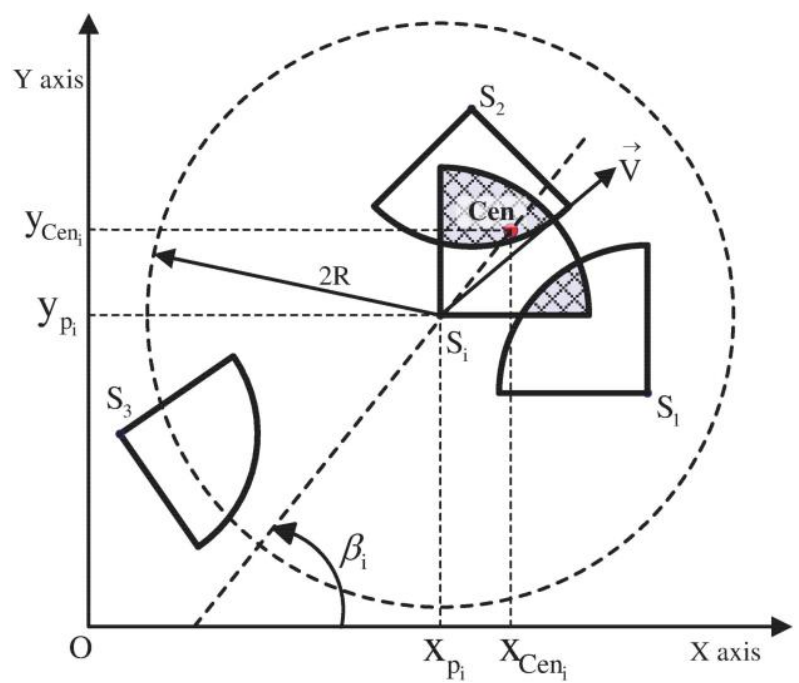

Fig 5: The Sensing model with neighboring sensors

\section{PROPOSED IMPLEMENTATION}

1. The segments for lower energy node consumption formulation \& 2 categories of important things to estimate the k-coverage probability in a network with log-normal investigation (though the shadowing distribution can be slightly random and without coverage sensing area of higher energy shifting nodes.

2. Sensors node is used quadrature technique or a modest analytic formula for coverage sense with advance node energy.

3. The new composite high-dimensional important uses quadrature approaches for low dimensions and quasi-random integration for higher $(n>2)$ computes 1-coverage probability for a network with Rayleigh fading (exponentially circulated with unit mean) and log-normal shadowing.

4. We present a modification of variables motivated by the dimensional spherical coordinates.

$\mathrm{s} 1=\mathrm{u}[\sin \theta 1 \sin \theta 2 \ldots \sin \theta \mathrm{n}-1] 2 / \beta$

$\mathrm{s} 2=\mathrm{u}[\cos \theta 1 \sin \theta 2 \ldots \sin \theta \mathrm{n}-1] 2 / \beta$

$\mathrm{s} 3=\mathrm{u} \cos \theta 2 \sin \theta 3 \ldots \sin \theta n-1] 2 / \beta \ldots$

$\mathrm{sn}=\mathrm{u}[\cos \theta \mathrm{n}-1] 2 / \beta$.

Where $q i=$ qi $(\theta \mathrm{i}, \ldots, \theta \mathrm{n}-1):=(\mathrm{si} / \mathrm{u}) \beta / 2$.

When $\beta=2$ our system of synchronizes boils down to the regular n-dimensional spherical coordinates, whose Jacobin is $\mathrm{J}^{-}(\mathrm{u}, \theta 1 \ldots \theta \mathrm{n}-1)=\mathrm{u} n-1 \mathrm{Qn}-1 \mathrm{i}=1$ sini-1 $\theta \mathrm{i}$; $\operatorname{cf}[21$, eq. (1.5)]). By overview (or element belongings and the chain rule) our coordinate system has the corresponding Jacobian Mathematical addition of hyper geometric function $2 \mathrm{~F} 1$ is used when the model has noise. A close-form answer with $2 \mathrm{~F} 1$ is used in the no noise case.

5. Simulation characters are also included for valuation determinations. All network base stations are tried on a disk region. The disk region wants to be large sufficient to decrease "edge effects", which become more protruding when fading is included.

We accept noise power $-96 \mathrm{dBm}$ standardized by the base station power $62.2 \mathrm{dBm}$ which types $\mathrm{W}=10-15.82$. We reflect two values for the density of base stations: $\lambda=$ $4.619 \mathrm{~km}-2$, which corresponds to a "OLSR" network deployment and $\lambda=0.144 \mathrm{~km}-2$ for a "suburban" one. The evaluation of the proposed algorithm showed that the previous approach is less reliable and more time-taking as compared to the I-ECOSR.

\subsection{Algorithm for I-ECOSR}

1. Find neighboring sensors nodes in grid ;

2. If dead node occurrences;

3. Shift advance node due to dead node in another grid or area;

4. Set parameter for state $=1$

5. While (overlap==1)

6. Calculate OSR;

7. The Nodes are overlap optimal angle according to the rotation angle function

8. if (network is equilibrium)

9. $\quad$ overlap $=0$;

10. end

11. end

12. calculate OSR;

13. while $(\mathrm{OSR}>=$ predefined threshold $)$

14. calculate priority;

15. if(priority is highest)

16. state $=0$;

17. send state information to its neighboring sensors

18. else

19. calculate OSR;

20. end

21. end

\section{RESULT}

The simulated result shows the performance of the improved (I-ECOSR) algorithm, the Random approach in which each sensor select its sensing direction randomly.

Table 1. Simulation parameter

\begin{tabular}{|l|l|}
\hline Simulation Parameters & Values \\
\hline Network of field size (area) & $\mathbf{2 0 0 * 2 0 0}$ \\
\hline Number of sensor nodes (N) & $\mathbf{1 0 0}$ \\
\hline
\end{tabular}




\begin{tabular}{|l|l|}
\hline $\begin{array}{l}\text { Number of advanced nodes } \\
\text { (an) }\end{array}$ & $\mathbf{0 . 2}$ \\
\hline Number of normal nodes(nn) & $\mathbf{0 . 8}$ \\
\hline Energy of a normal node $\left(\mathrm{E}_{0}\right)$ & $\mathbf{0 . 5}$ \\
\hline Location of the base station & Centroid \\
\hline $\begin{array}{l}\text { Sensor network deployment } \\
\text { type }\end{array}$ & Random \\
\hline Simulator software Version & $\mathbf{2 0 1 2 a}$ \\
\hline Mobility model & Random wave-point \\
\hline Sensing range & $\mathbf{1 0 0}$ \\
\hline Grid radius & $\mathbf{3 . 5}$ \\
\hline Fading & AWGN \\
\hline
\end{tabular}

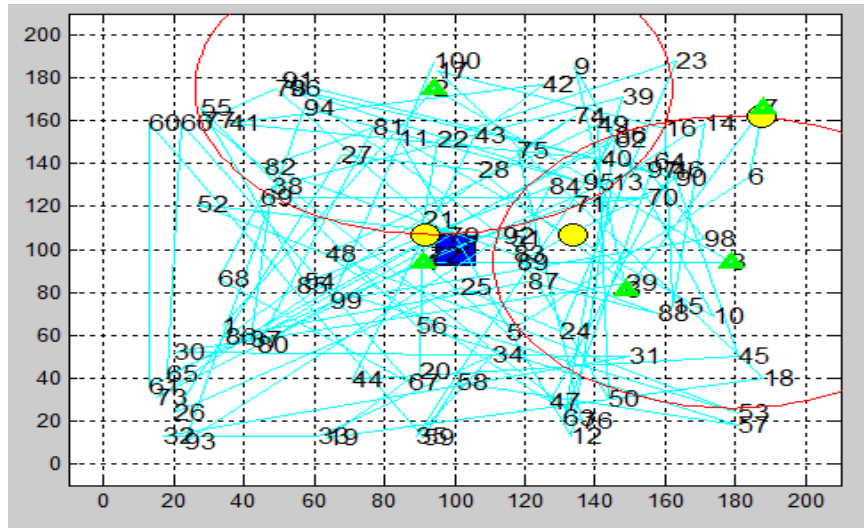

Fig. 7: nodes shifting towards the minimum energy node in other grid

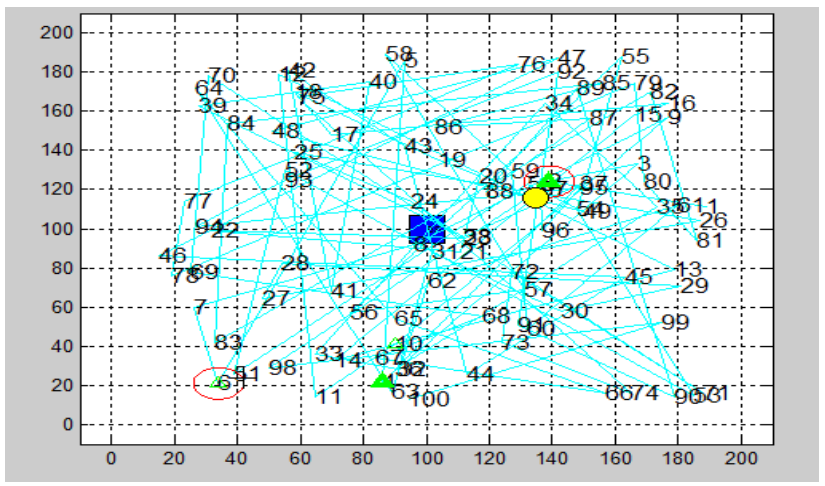

Fig. 8: After shifting the nodes, network update the nodes position of nodes

In below figure 9, the coverage ratio of I-ECOSR getting improvised with respect to time-step at initial stage gaining better improvement when coverage increasing in response time as compare to Enhanced Coverage Ratio (ECRM). Earlier methods implemented had the problem that the sensing range of the advanced node falls short while shifting towards the low energy node. So in I-ECOSR, the sensing range of nodes is improved by reducing the energy consumption of advanced nodes in order to enhance the coverage ratio, hereby providing the increased efficiency and reliability of the network.

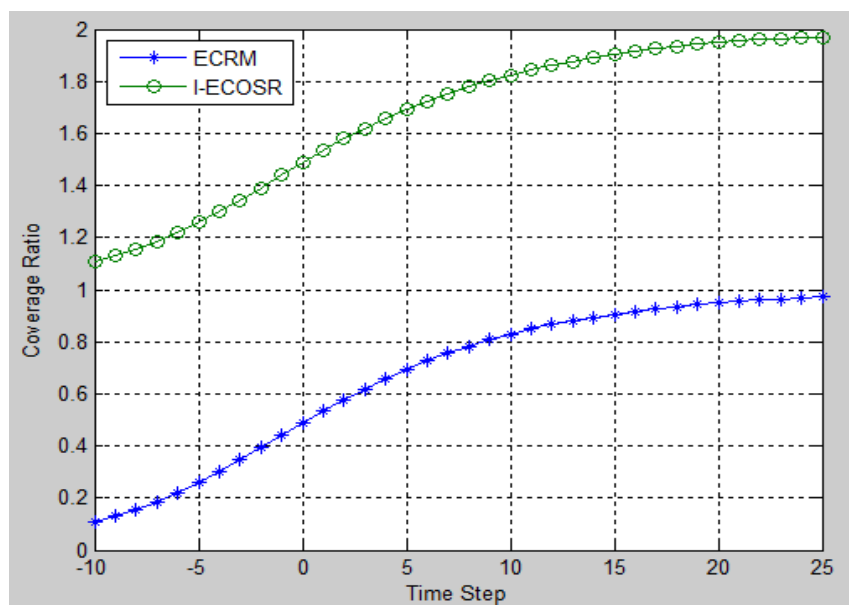

Fig. 9: Improved Coverage ratio for I-ECOSR comparatively to Energy Coverage ratio (ECRM) 


\section{CONCLUSION}

In this paper, a grid based enhance coverage ratio with overlap sensing related to connected coverage in directional wireless sensor networks is acquired. Specifically, it investigated the dead node problem. The Improved enhance coverage overlap sensing ratio (I-ECOSR) approach is proposed, which is based on the size of overlapped area between directional sensors. The comparison is made between the proposed scheme with the ECRM and Simulation results showed that I-ECOSR outperform for the coverage enhancement \& overcome the dead node occurrence, thus increasing the stability and efficiency of the network.

Future work will focus on merging the algorithm with an energy consumption model to give deliberation to both coverage and lifetime performance in mobile and directionrotatable directional sensor networks.

\section{REFERENCES}

[1] Friis, H.T.: A Note on a Simple Transmission Formula. In Proceedings of the IRE, Vol. 34, No. 5, (1946) 254256 2. He, T., Huang, C., Blum, B. M., Stankovic, J. A., and Abdelzaher, T.: Range-free localization schemes for large scale sensor networks.

[2] Y. Mohamed and K. Akkaya, "Strategies and techniques for node placement in wireless sensor networks: A survey," Ad Hoc Networks, vol. 6, no. 4, pp. 621-655, 2008.

[3] Kansal, W. Kaiser, G. Pottie, M. Srivastava, and G. Sukhatme, "Reconfiguration methods for mobile sensor networks," ACM Transactions on Sensor Networks, vol. 3, no. 4, 2007.

[4] M. A. Guvensan and A. G. Yavuz, "On coverage issues in directional sensor networks :A survey," Elsevier AdHoc Network s , February 2011.
[5] D. Tao, H. Ma, and L. Liu, "Coverage-enhancing algorithm for directional sensor networks," in Lecture Notes in Computer Science:Mobile Ad-hoc and Sensor Networks, vol. 4325, November 2006, pp. 256-267.

[6] J. Zhao and J.-C. Zeng, "An electrostatic field-based coverage-enhancing algorithm for wireless nmultimedia sensor networks," in Proc. of IEEE Intl. Conf. on Wireless Communications, Networking and Mobile Computing (WiCom '09),Beijing, China, September 2009, pp. 1-5.

[7] H. Ma, X. Zhang, and A. Ming, "A coverage-enhancing method for 3d directional sensor networks,"in Proc. Of IEEE Intl. Conf. on ComputerCommunications(INFOCOM'09), Rio de Janerio, Brazil, April 2009, pp. 2791-2795.

[8] L., Adler, R., Buonadonna, P., Chhabra, J., Flanigan, M., Kushalnagar, N., Nachman, L., and Yarvis, M.: Design and deployment of industrial sensor networks: experiences from a semiconductor plant and the North Sea. In Proceedings of the 3rd international conference on Embedded networked sensor systems . (2014) 64-75

[9] Rajagopalan, Ramesh and Varshney, Pramod K., "Data aggregation techniques in sensor networks: A survey" (2006). Electrical Engineering and Computer Science. Paper 22.

[10] Neng-Chung Wang, Yung-Kuei Chiang, Chih-Hung Hsieh, and Young-Long Chen : Grid-Based Data Aggregation for Wireless Sensor Networks in Journal of Advances in Computer Networks, Vol. 1, No. 4, December 2013 